\title{
Augmentation of transgene-encoded protein after neonatal injection of adeno-associated virus improves hepatic copy number without immune responses
}

\author{
Denise S. Tai', Chuhong Hu' , Elizabeth H. Kim' and Gerald S. Lipshutz ${ }^{1-7}$
}

BACKGROUND: Achieving persistent expression is a prerequisite for genetic therapies for inherited metabolic enzymopathies. Such disorders potentially could be treated with gene therapy shortly after birth to prevent pathology. However, rapid cell turnover leads to hepatic episomal vector loss, which diminishes effectiveness. The current studies assessed whether tolerance to transgene proteins expressed in the neonatal period is durable and if the expression may be augmented with subsequent adeno-associated virus (AAV) administration. METHODS: AAV was administered to mice on day 2 with reinjection at 14 or at 14 and $42 \mathrm{~d}$ with examination of changes in hepatic copies and B and T cell-mediated immune responses.

RESULTS: Immune responses to the transgene protein and AAV were absent after neonatal administration. Reinjection at 14 or at 14 and $42 \mathrm{~d}$ resulted in augmented expression with greater hepatic genome copies. Unlike controls, immune responses to transgene proteins were not detected in animals injected as neonates and subsequently. However, while no immune response developed after neonatal administration, anticapsid immune responses developed with further injections suggesting immunological ignorance was the initial mechanism of unresponsiveness.

CONCLUSIONS: Persistence of transgene protein allows for tolerance induction permitting readministration of AAV to reestablish protein levels that decline with growth.

$I_{s}^{n}$ $\mathrm{n}$ individuals with genetic diseases of abnormal protein synthesis, the normal protein may be recognized as a neoantigen leading to a potential immune reaction with the early introduction and expression by gene transfer (1-3). The likelihood of an immune response to an expressed protein is influenced by several factors including the specific host, the underlying mutation in the protein, the type of gene delivery vector, and the route by which the vector is administered (3). In animal models, xenogenic homologous proteins are more immunogenic than are proteins from the same species (2-4). In addition, the tissue in which genes are expressed may affect the likelihood of eliciting immune responses $(3,5)$.

We have demonstrated that early expression is detected in neonatal mice with different adeno-associated virus (AAV) serotypes; some, such as serotype 9 and rh10 have improved vector properties such as higher transduction efficiencies (6-7). Such early administration after birth results in persistent gene expression that can be achieved after a single dose (6-8). The serotype and cell cycle of the tissue of interest (e.g., liver vs. muscle) (8) may determine whether substantial persistent expression remains as cells and tissues grow and divide in this period of rapid cellular proliferation of the neonate; hepatic loss of episomal AAV results in a substantial expression decline in mice during the first several weeks of life (6) and this loss can affect the efficacy of therapy $(7,9)$. Such findings demonstrate the challenges that rapid cellular proliferation adds to treatment initiated early in life with episomally-located vector genomes.

In adult mammals, readministration of the same serotype of AAV is generally not successful due to neutralizing antibody responses to the viral capsid proteins (10-13) that develop after the initial administration. However, delivery of geneexpression vectors in a mammal where the immune system is immature may facilitate the development of tolerance to therapeutic proteins (14). In utero and neonatal gene transfer have the potential for preventing the development of disease and may allow for transduction of expanding stem cell populations or organ systems that may not be accessible postnatally (15-16). In previous studies, we have been able to administer AAV expressing factor VIII during the neonatal period (7). This led to operational tolerance to this antigen. However, the decline in transgene-encoded protein expression, particularly during the early rapid growth phase of dividing tissues of neonatal and juvenile mice, remains a substantial problem that affects the long-term high-level protein expression that may be necessary for correcting certain genetic disorders affecting

\footnotetext{
The first two authors contributed equally to this work.

'Department of Surgery, David Geffen School of Medicine at UCLA, Los Angeles, California; ${ }^{2}$ Department of Medicine, David Geffen School of Medicine at UCLA, Los Angeles, California; ${ }^{3}$ Department of Psychiatry, David Geffen School of Medicine at UCLA, Los Angeles, California; ${ }^{4}$ Department of Urology, David Geffen School of Medicine at UCLA, Los Angeles, California; Intellectual and Developmental Disabilities Research Center at UCLA, David Geffen School of Medicine at UCLA, Los Angeles, California; ${ }^{6}$ Broad Center for

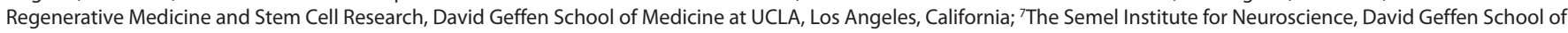
Medicine at UCLA, Los Angeles, California. Correspondence: Gerald S. Lipshutz (glipshutz@mednet.ucla.edu)
} 
the liver (8-9). Similar growth, albeit at a slower rate, over a longer period of time is present in humans. Newborns typically double their body weight in the first months of life and triple it within the first year (17); the human liver has similar increases in size: first doubling by $3 \mathrm{mo}$, a second doubling by $10 \mathrm{mo}$, and a doubling again by about year 5 (18). The focus of the present studies was to assess the durability of operational tolerance with neonatal delivery of AAV and expression of a xenogenic transgene-encoded protein and if augmentation of hepatic expression and genome copy number was possible with subsequent AAV administration.

\section{RESULTS}

\section{Augmenting Expression With Postnatal Doses of AAV}

In these experiments, all mice were administered $3 \times 10^{12} \mathrm{gc} / \mathrm{kg}$ of AAV on the second day of life (Figure 1a). The first group of animals ( $n=5$ per time point) received a vector injection as a single dose. Subsequently, a second group of mice $(n=5$ per time point) received the same serotype vector, rh10, on day 2 and day 14 of life with genome copy number of AAV and expression along with immune responses examined. A final group ( $n=5$ per time point) received AAV serotype rh10 on the second day of life and at $2 \mathrm{wk}$ and then received a further augmenting dose at 6 wk of life, this time with serotype switched AAV9. All animals received AAV with the CBA promoter/CMV enhancer and firefly luciferase. To determine an optimal time for readministration of vector, the kinetics of murine liver growth was examined (Figure 1b). By $6 \mathrm{wk}$, the liver had undergone nearly four doublings since birth with the mouse itself reaching nearly adult size.

Administration of AAV to neonatal mice was well tolerated and resulted in early expression of luciferase; peak expression a

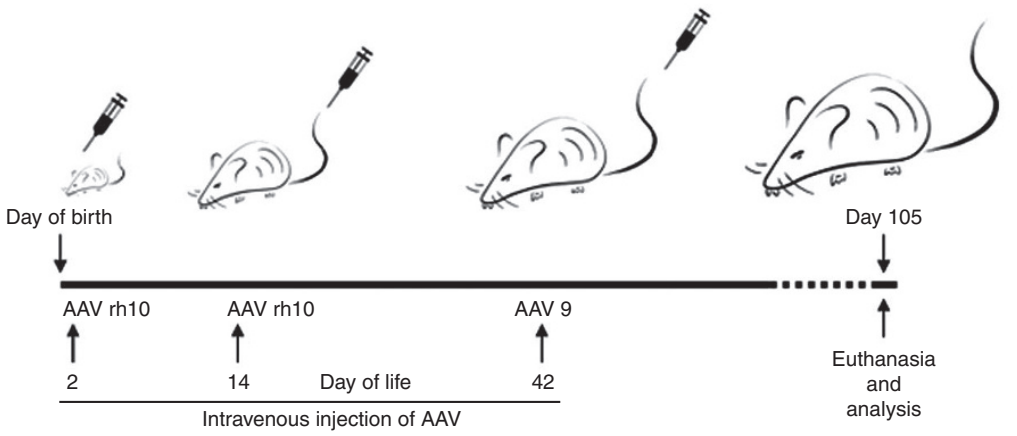

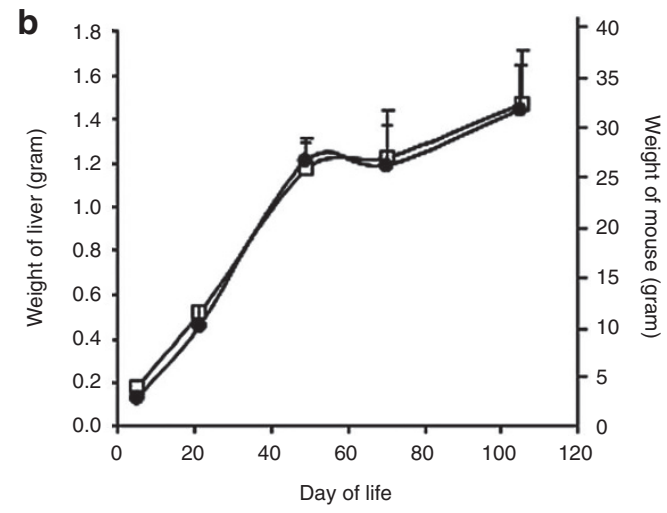

C

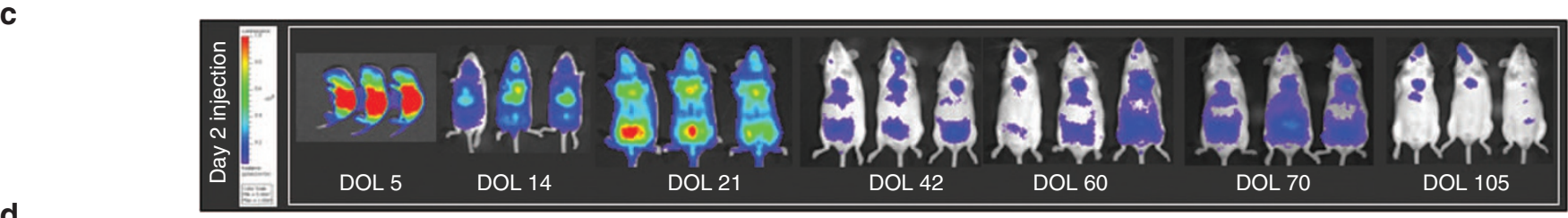

d
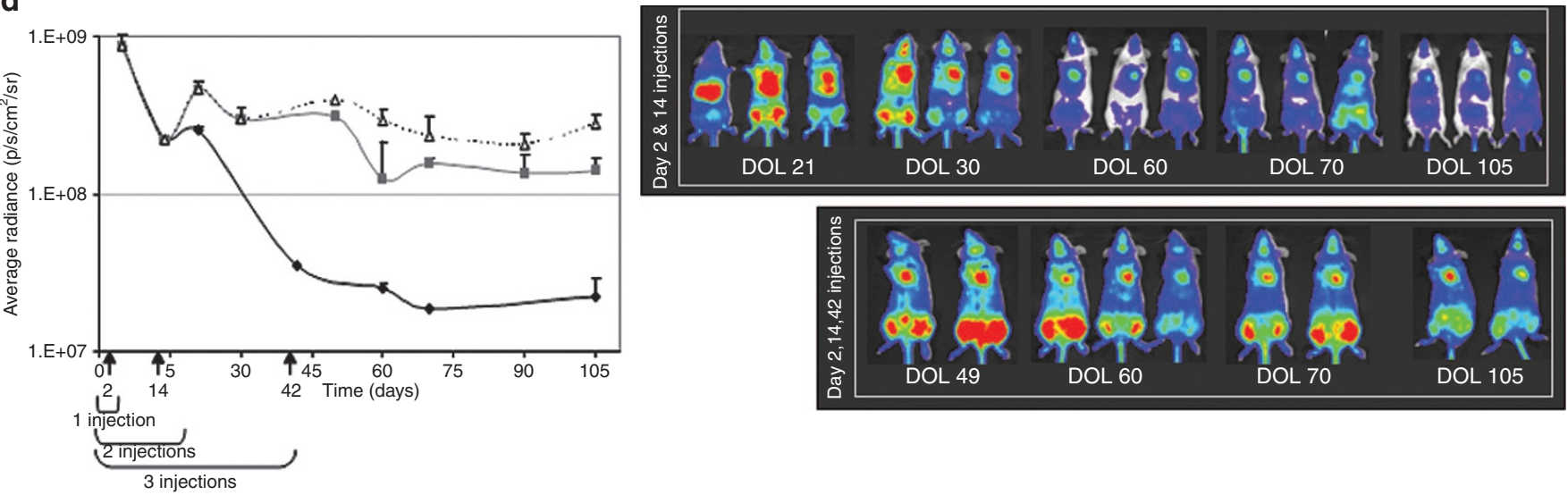

Figure 1. Change in transgene expression with subsequent AAV administration. (a) Schematic of experiments. (b) Increase in weight of mouse and liver with time. Mice grow most rapidly during the first 5-6 wk of life after which the rate of weight gain slows. During this period, mouse and liver weight increases fourfold ( liver weight; $\square$ animal weight). (c) In vivo imaging of firefly luciferase after intravenous injection of adeno-associated virus (AAV) on the second day of life and additional injections with subsequent photon diffusion patterns. First group: Neonatal mice were injected a single time with rh10 serotype AAV. Second group: Mice were injected twice with rh10 serotype AAV, first as neonates on the second day of life, followed by an intravenous injection on day 14. Third group: Mice were injected three times with AAV with rh10 on the second day of life and day 14 followed by AAV 9 on day 42 . All mice were followed for $15 \mathrm{wk}$. The same pseudocolor scale was used for each of the animals, with blue indicating lower levels and red indicating higher levels of luciferase activity; thus direct comparison can be made. (d) Quantitation of photon emission as luciferase gene expression ( $\bullet$ injection, $\mathbf{\square} 2$ injections, $\Delta 3$ injections). Data are expressed as mean + SD. Mouse and syringe drawings: $\odot$ Fotosearch.com, reprinted with permission. DOL, day of life. 
was detected at the first time point, $72 \mathrm{~h}$ after vector administration (Figure 1c, day of life 5). Mice were followed longitudinally using bioluminescent imaging. Mice that received the single dose of AAV as a neonate had a substantial loss of expression during the $15 \mathrm{wk}$ of study (Figure 1c,d). Expression was widely distributed with use of the CBA promoter/CMV enhancer up to week 3 of life, but then expression markedly declined with residual expression primarily in the heart and lungs at the end of the study (Figure 2). This corresponded with a marked decline of AAV genome copies in the liver by nearly 3 logs to about $0.2 \%$ of the copy number 15 wk after vector administration in the neonatal period (Table 1).

Because of the vector copy number decline, we decided to initially administer vector at day 2 of life followed by an augmenting dose at $2 \mathrm{wk}$ of age, suspecting there would still be substantial loss of AAV vector genomes due to continued hepatocellular division. After the augmenting dose of AAV at $2 \mathrm{wk}$, expression was maintained at higher levels longer than the group of mice that received only one dose of AAV on day 2 (Figure 1c, day 2 and 14 injections). In the augmented group, higher residual expression was found at week 15 in multiple tissues: the heart, liver, and lung (Figure 1c). With the single administration of AAV on the second day of life, there were $1.3 \mathrm{gc} / \mathrm{ng}$ DNA at $15 \mathrm{wk}$ in the liver (Table 1 ). While there is variability due to some differences in size of animals at the 2-wk injection, with this second dose the residual AAV hepatic copies at $15 \mathrm{wk}$ had increased on average eight times to $10.4 \mathrm{gc} / \mathrm{ng}$ DNA.

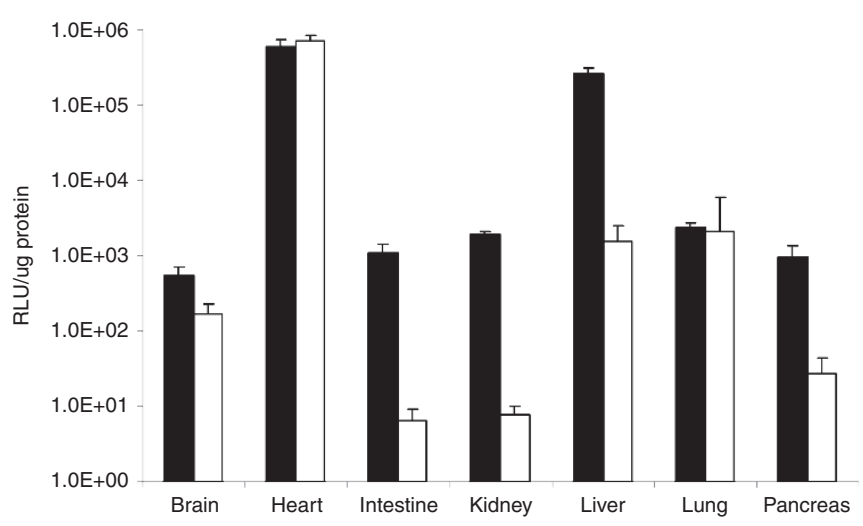

Figure 2. Levels of adeno-associated virus-mediated tissue gene expression decline with animal growth. Tissues were removed from animals on day $5(\square)$ and day $105(\square)$ and gene expression levels were measured and normalized to protein content. All tissues examined demonstrated declines in expression except for the heart. Data are expressed as mean + SEM.
Because the adult size of the liver is nearly attained by $6 \mathrm{wk}$ of age, we decided to give an additional dose of AAV at that time to determine if expression could be further stabilized. Administration of this third dose of vector resulted in higher levels of expression in multiple tissues (Figure 1c, day 2, 14, and 42 injections) and with higher sustained expression at these levels to at least $15 \mathrm{wk}$ of life. Hepatic genome copy numbers, again affected by some variability of sizes when injected, were higher at $15 \mathrm{wk}$ ( $29.3 \mathrm{gc} / \mathrm{ng}$ DNA): on average 22.5 times that of the single dose at day 2 and 2.8 times that of mice having received AAV on the second and fourteenth days of life.

\section{The Role of Innate Immunity in Viral Copy Number Decline}

While the decline of copies of AAV with hepatocellular division has been previously described by our group $(6,8)$ and others (19-20), the mechanism is not completely understood. To determine if the innate immune system has any role in this decline, we performed studies in the MyD88 knockout mouse. Toll-like receptor-mediated responses are important in the innate immune response to certain viral infections. The MyD88 gene is essential for their control and the maturation and activation of virus-specific $\mathrm{CD}^{+}(21)$ and the regulation of virus-specific $\mathrm{CD}^{+}(22) \mathrm{T}$ cells. We hypothesized that if innate immunity had a role in the decline of AAV copy number in the liver then we would have higher numbers of AAV in hepatocytes of the MyD88 knockout animals after the administration of AAV on the second day of life.

MyD88 $(n=4)$ and control mice $(n=5)$ received identical doses of AAVrh10 on the second day of life. Both groups were euthanized at $35 \mathrm{~d}$. Livers were removed and viral DNA was quantified using quantitative polymerase chain reaction to determine total viral copy number per genomic DNA. At day 35, both MyD88 and wild-type mice demonstrated similar residual genome copy numbers suggesting that innate immunity and activation of virus-specific T cells are likely not involved in the decline in copy number with hepatocellular division (Figure 3). Differences in copy numbers are likely due to strain differences. The MyD88 mice were obtained on the $\mathrm{C} 57 \mathrm{Bl} / 6$ background and thus control studies were also performed with $\mathrm{C} 57 \mathrm{Bl} / 6$; $\mathrm{FVB} / \mathrm{N}$ mice were used for all other studies.

\section{Cellular Immune Responses to Transgene-Encoded Protein}

To examine if cellular immunity develops to transgeneencoded proteins when expression begins in the neonatal period, we employed an enzyme-linked immunospot (ELISPOT) assay (Figure 4). The ELISPOT technique is useful

Table 1. Change in genome copy number in liver with injection

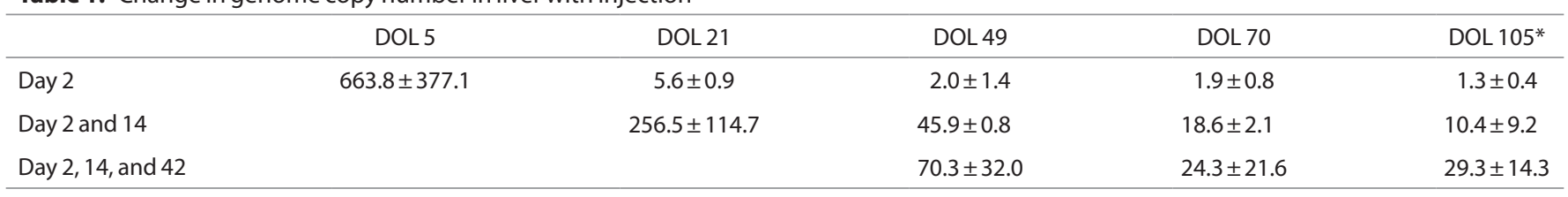

Numbers are expressed as genome copies per nanogram DNA.

${ }^{*} P=0.003$. 


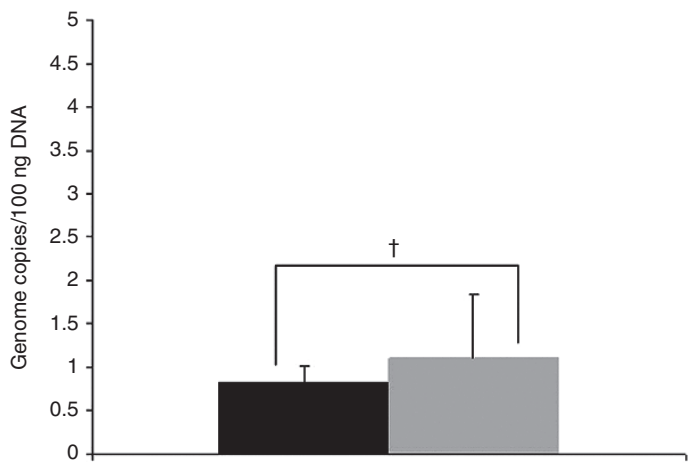

Figure 3. Genome copy number decline is not affected by innate immunity. Vector copy number was measured in the liver of MyD88 mice by quantitative polymerase chain reaction at day 35 after adeno-associated virus administration $\left(3 \times 10^{12} \mathrm{gc} / \mathrm{kg}\right.$ rh10 luciferase) on day 2 of life. (Data are expressed at mean + SD. $\square$ designates wild-type liver and designates liver from MyD88 knockout. ${ }^{\dagger} P=0.5$.

to both qualitatively and quantitatively monitor cell-mediated immunity as it is sensitive and accurate in the detection of rare antigen-specific T cells. In these studies, IL-2 (involved in adaptive immunity by augmenting T-cell proliferation, survival, and effector differentiation) (23) and IFN- $\gamma$ (a Th1 cytokine where $\mathrm{T}$ lymphocytes are the major source in the adaptive immune response) (24) were examined.

Three groups of mice were studied: (i) positive controls $(n=4)$ that received saline intravenously on the second day of life followed by luciferase with adjuvant at day 35; (ii) negative controls that received saline intravenously on day 2 and on day 35; and (iii) an experimental group $(n=4)$ that received AAV expressing luciferase on day 2 followed by luciferase with adjuvant on day 35 . Splenocytes were collected after the vaccinating dose and examined by ELISPOT stimulated with recombinant luciferase.

Cells were plated and spots were examined with recombinant luciferase used at $0.5 \mu \mathrm{g}$ per well and the number of IL-2 and IFN- $\gamma$ spots elicited from splenocytes were studied. While all groups did respond to ConA, negative control animals (those that received saline neonatally and no recombinant luciferase/ adjuvant postnatally) as expected did not substantially demonstrate either IL-2-secreting or IFN- $\gamma$-secreting lymphocytes. Also as expected, the positive control animals, which were naive to luciferase before vaccination, had development of both IL-2- and IFN- $\gamma$-secreting splenocytes. The experimental group had results similar to that of the negative controls; that is adult mice that were administered AAV-expressing luciferase in the neonatal period did not have production of IL-2 or IFN$\gamma$ from splenocytes after vaccination.

\section{Humoral Immune Responses to AAV Capsid Proteins}

We have previously shown that humoral immune responses do not develop to AAV capsid proteins after a neonatal dose of IV-administered AAV. The durability of this unresponsiveness has not been clear. In these studies, we examined the humoral immune response to AAV with subsequent vector administration in mice that received AAV in the neonatal period.

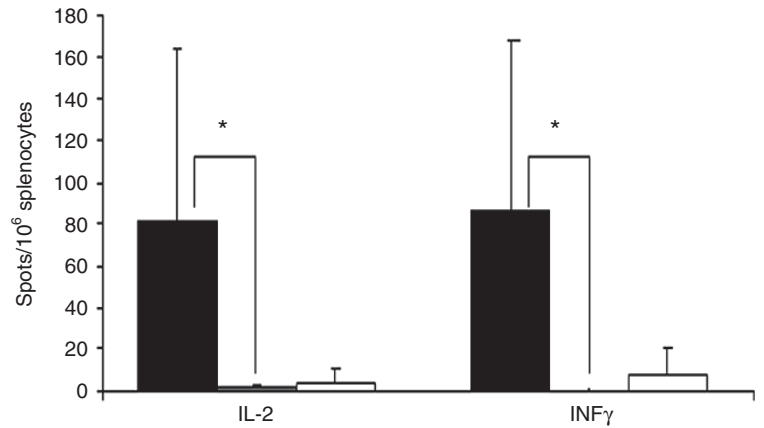

Figure 4. ELISpot data demonstrate an absence of cell-mediated immune responses against luciferase after neonatal injection. Adult splenocytes were stimulated with luciferase to examine for a proliferative response after neonatal injection of AAVrh10-luciferase. Negative controls ( $\square$ ) (DOL 2 saline $\rightarrow$ DOL 35 saline) included neonatal animals not exposed to luciferase protein in vivo. Positive controls $(\square)$ were animals that received saline only as a neonate followed by luciferase/adjuvant at 5 wk. Experimental animals $(\square)$ received adeno-associated virus (AAV)-luciferase as a neonate followed by luciferase/adjuvant at $5 \mathrm{wk}$. Data are presented as mean + SD. ${ }^{*} P \leq 0.05$.

Using a serotype-specific enzyme-linked immunosorbent assay (ELISA), plasma of mice were examined at day 14 after day 2 administration of AAVrh10. As previously demonstrated (7), there was no development of antibody to serotype rh10 (Figure 5a (white bar)). Because of the lack of development of anticapsid humoral immunity, administration of a second dose of AAVrh10 on day 14 was possible. However, when measured at day 42 , high levels of antibody to serotype rh10 developed 4 wk after this second administration (black bar, Figure 5a), suggesting that operational tolerance did not develop to AAVrh 10 capsid ( $n=3-5$ per group).

The humoral immunity that developed was found to be capsid specific. After two injections (day 2 and day 14) of AAVrh10, antibody did not develop to AAV9 (Figure 5b, white and black). However, after the administration of AAV9 at $6 \mathrm{wk}$, antigen-specific humoral immunity to AAV9 also developed (Figure $5 b$, gray bar) ( $n=3-5$ per group).

In control naive juvenile or adult animals when AAVrh10 was administered at $2 \mathrm{wk}$ and AAV9 was administered at $6 \mathrm{wk}$, capsid-specific antibodies developed to both AAVrh10 and AAV9 (Figure 5a,b gray hatched bars) $(n=5)$. However, in the experimental mice, antigen-specific operational tolerance to AAVrh10 administered in the neonatal period did not result. While a second dose at day 14 did allow for repeat serotype rh10 transduction, serotype switching for further transgene augmentation was necessary at $6 \mathrm{wk}$ due to the interval development of capsidspecific humoral immunity to serotype $\operatorname{rh} 10$ ( $n=5$ per group).

\section{Humoral Immune Response to Transgene-Encoded Protein}

We have previously demonstrated that a humoral immune response to transgene-encoded proteins could be avoided with a single neonatally administered dose of AAV expressing a transgene in mice (7). The durability of this lack of immune response has not been previously evaluated to immunogenic and xenogenic proteins such as luciferase. Neonatal mice on day 2 of life were administered AAVrh10 expressing luciferase 


\section{Tolerance to transgene-encoded protein}

a

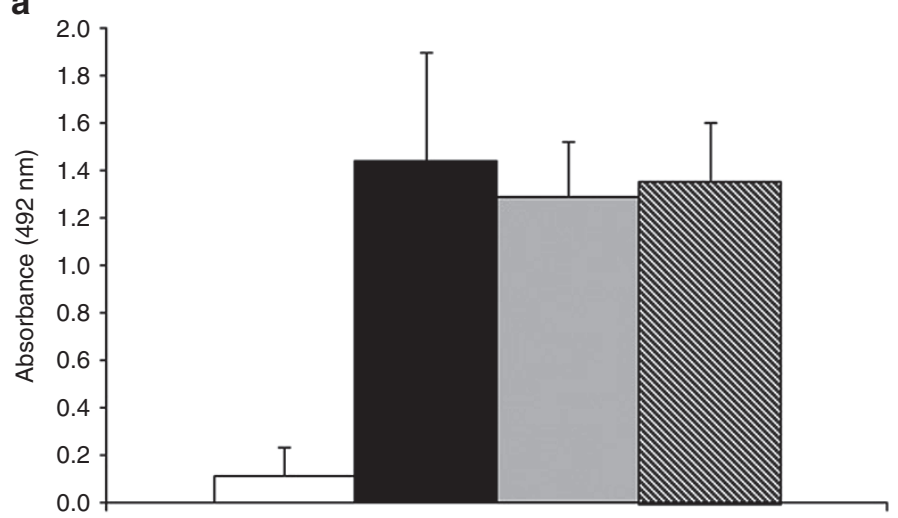

b

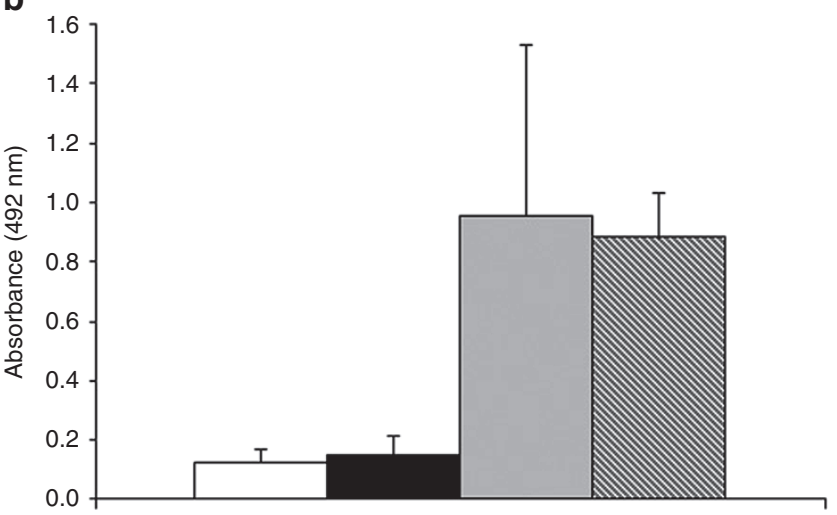

Figure 5. Absence of antibody-mediated responses against adeno-associated virus (AAV) after neonatal administration. (a) Antibody titers to AAV rh10 capsid proteins were measured (as adults) by enzyme-linked immunosorbent assay (ELISA) after neonatal injection of AAVrh10 at day 2 of life ( $\square$ ), at day 2 and day $14(\square)$, and at day 2 and day 14 with rh 10 and with AAV serotype 9 at day 42 ( $\square$ ). Positive control was demonstrated by administering AAVrh 10 on day 14 and AAV 9 on day 42 ( $\mathbb{\nabla}$ ). (b) Antibody titers to AAV 9 capsid proteins were measured by ELISA after neonatal injection of AAVrh10 at day 2 of life $(\square)$, at day 2 and day $14(\square)$, and at day 2 and day 14 with rh10 and with AAV serotype 9 at day 42 ( $\square$ ). Positive control was demonstrated by administering AAVrh10 on day 14 and AAV 9 on day 42 (畂. Plasma was collected at least 14 days after AAV administration for both sets of studies. (Data are presented as mean $+\mathrm{SD}$.)

( $n=5$ per group). Subsequent injections were given at 2 and at $6 \mathrm{wk}$. In both cases, antibodies did not develop to the luciferase protein (Figure 6, first 3 bars). Conversely, when naive animals were administered AAV expressing luciferase at 2 and $6 \mathrm{wk}$ of life, a strong antigen-specific humoral immune response did develop (Figure 6, 4th bar).

To further test the durability of this operational tolerance, vaccination was performed with luciferase and adjuvant. Experimental mice were administered AAV rh10 expressing luciferase on the second day of life while control animals received saline. Plasma was collected on day 34 from both groups followed by vaccination the next day. Plasma was then collected on day 70 from both groups and analyzed by a luciferase-specific ELISA. While control animals, as expected, demonstrated high titers of undilutable antibody to at least 1:2,560 (Figure 7, dotted bars), the experimental animals had a substantially blunted humoral immune response that was reduced with dilution (Figure 7, gray bars).

\section{DISCUSSION}

The present studies demonstrate that (i) neonatal delivery of AAV produces long-term transgene-encoded protein expression without the development of cellular or humoral immunity to either virus or gene product (suggesting that operational tolerance to the stably expressed foreign protein, but not to transiently present capsid, can be achieved); (ii) delivery of a second postnatal dose of AAV results in a humoral immune response to AAV capsid proteins, which suggests that immunologic ignorance was the mechanism responsible for the unresponsiveness seen after neonatal administration; (iii) the innate immune system does not appear to influence the decline in AAV copy number in hepatocytes after neonatal administration; and (iv) transgene expression and AAV hepatic copy number can be augmented postnatally by readministration of the same or a different serotype AAV vector since operational tolerance was established to the transgene-encoded protein.

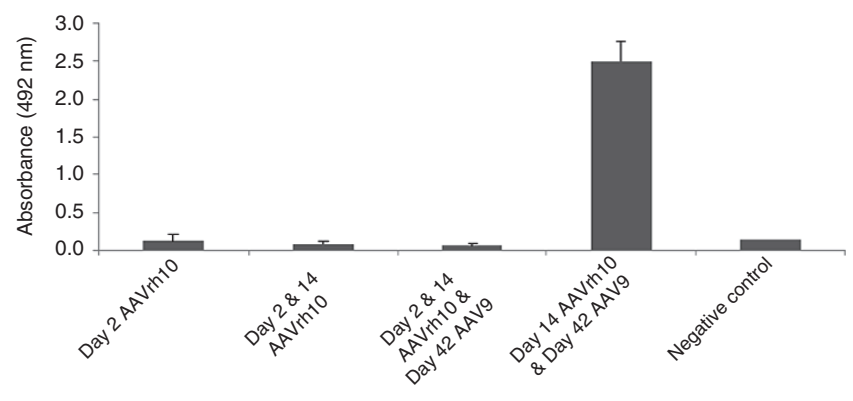

Figure 6. Absence of antibody-mediated responses against luciferase after neonatal and subsequent injections for augmentation of expression and hepatic copy number. Antibody titers to luciferase were measured by enzyme-linked immunosorbent assay after neonatal injection of AAVrh10luciferase at day 2, after injections at day 2 and day 14, and with a subsequent dose of AAVg at $42 \mathrm{~d}$. Control studies included animals where adeno-associated virus (AAV) expressing luciferase was administered at 14 and $42 \mathrm{~d}$. Data are presented as mean + SD.

There are two major mechanisms reported to prevent the reactivity of $\mathrm{CD} 8+\mathrm{T}$ cells: ignorance and tolerance. When ignorance is operative, naive autoreactive $\mathrm{CD} 8+\mathrm{T}$ cells ignore antigens and recirculate without causing damage. In the case of tolerance, $\mathrm{CD} 8+\mathrm{T}$ cells are deleted if the mechanism is centrally mediated or controlled by $\mathrm{T}$ regulatory cells if the mechanism is peripheral. Which factors contribute to each particular outcome is only partly known. When antigen is expressed and/or cross-presented at concentrations too low to stimulate $\mathrm{T}$ cells, peripheral $\mathrm{T}$ cells can remain "ignorant" of the antigen (25). Thus ignorant $\mathrm{T}$ cells, unlike tolerant $\mathrm{T}$ cells, are not rendered dysfunctional from future antigen encounters but remain antigen inexperienced: they persist as naïve but potentially functional and can be activated by external stimuli.

A number of studies have suggested that the relative immunological immaturity of the fetus (and likely the neonatal mouse) may contribute to diminished immune responses or induction of immune tolerance (14). Before thymic processing 


\section{Articles | Taietal.}

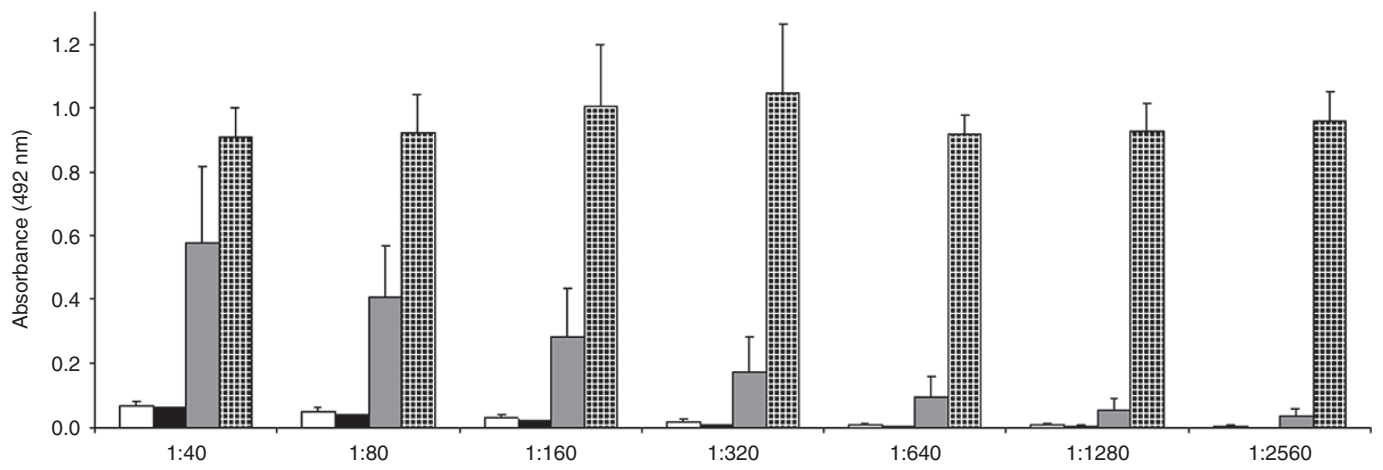

Figure 7. Blunted humoral immune response to luciferase in neonatally injected animals after stimulating adult animals with purified luciferase and adjuvant. 35-d-old mice were administered luciferase/adjuvant after having received AAVrh10-luciferase ( $\square$ ) or saline ( $\square$ ) as neonates. Plasma was examined for antiluciferase antibodies at day 34 (before adult injection) and at day 70 (after luciferase and adjuvant injection intraperitoneally) in mice that received adeno-associated virus (AAV) as neonates ( $\square$ ) and those that received saline as ( $-\dot{-})$ neonates. The x-axis indicates plasma dilution; the y-axis demonstrates the optical density of samples analyzed by spectrophotometry. Data are presented as mean + SD.

of lymphocytes in early immunologic development, induction of tolerance in the fetus to foreign proteins can occur. In the neonatal mouse the mechanisms may be different. Neonatal mice have a decreased frequency of professional antigen presenting cells including activated macrophages, B cells, and dendritic cells and murine neonates contain fewer $\mathrm{T}$ cells in their spleens (26-27); their T cells are functionally deficient in both in vivo and in vitro standard activation conditions. In addition, $\mathrm{T}_{\mathrm{H}} 1$ and cytotoxic $\mathrm{T}$ lymphocyte functions are poor with $\mathrm{T}_{\mathrm{H}} 2$ responses predominating (26). Other neonatal mouse studies suggest the expression of CD40 ligand is reduced in $\mathrm{T}$ cells (28) and the diversity of T-cell receptors is restricted (29). Additional evidence suggests that dendritic cell function may be immature resulting in a bias toward $\mathrm{T}_{\mathrm{H}} 2$ rather than $\mathrm{T}_{\mathrm{H}} 1$ responses $(3,30)$. These differences and the nature of the antigen presenting cells itself may determine whether the outcome of antigen presentation is neonatal tolerance or immunization (28).

In the studies conducted here, operational tolerance does not develop to AAV capsid protein. As the AAV vectors administered are replication incompetent, there are no coding genes for the capsid proteins. Thus, there is a transient presence of capsid that is lost after cellular transduction. However with subsequent administration of $\mathrm{AAV}$, anticapsid humoral immunity develops as it would in a naive immune system thus demonstrating the functionality of these cells. This lack of an immune response with initial AAV exposure appears to be consistent with immunological ignorance as functional immune cells were activated with later antigen exposure.

A number of studies have demonstrated that continual antigen exposure is required to maintain tolerance (31-33). In our present neonatal and prior in utero studies, luciferase expression was detected within $3 \mathrm{~d}$ of AAV administration and persisted for the lifetime of the animals $(7,34)$ without anti-AAV or antiluciferase humoral immunity. Because of the lack of an immune response to the transgene-encoded protein and viral vector, $\mathrm{AAV}$ in the present studies could be readministered and transgene-encoded protein expression, in this case luciferase, could be augmented as well as the genome copy number per hepatocyte. The establishment of operational tolerance allowed for the achievement of greater AAV copy number in juvenile and adult mice. Unlike neonates, where the rate of hepatocellular proliferation is much higher and affects episomal vector genomes (20), rapid cellular proliferation in adults is uncommon as individual hepatocytes in the mouse liver are replaced once every 180-400 d (35-36) suggesting that once an adult sized liver is attained, the hepatic copy number would be relatively stable with a slow decline over years. Thus, subsequent administration is most effective when adult liver size is reached but augmenting doses during the juvenile period are also of benefit. These doses could address the loss of hepatocyte copy numbers in infants treated with AAV for inherited metabolic disorders of the liver as they grow into childhood, adolescence, and later adulthood.

In utero injection of recombinant adenoviral vectors to murine fetuses also have been found to not elicit immune responses to adenovirus or luciferase in animals examined postnatally (37); however, gene expression was transient and no longer detected $4 \mathrm{wk}$ after birth. Thus while subsequent readministration of adenovirus in adult animals resulted in brisk humoral immune responses to adenoviral capsid proteins as expected, the animals also developed a strong humoral immune response to luciferase. It appears that this loss of transgene-encoded protein expression after neonatal administration resulted in the transgene-encoded protein subsequently being detected as a neoantigen by the immune system when later expression occurred with adult adenoviral vector delivery.

The ability to readminister AAV in mice injected with vector in the first few days of life appears to be due to ignorance, which allows for augmentation of expression postnatally. This could be important in certain disorders (e.g., hemophilia, Pompe disease) if therapeutic levels of protein expression were not maintained after neonatal administration. Alternatively, it could allow for the exogenous administration of recombinant protein (e.g., factor VIII or factor IX) without the development of inhibitory antibodies as operational tolerance has been achieved. The efficacy and safety of AAV delivered neonatally in mice provides an opportunity to develop strategies for the 
induction of tolerance to therapeutic proteins in humans. Furthermore in disorders where treatment with AAV was initiated in the neonatal period, repeated AAV dosing provides a method to augment the number of AAV vector genomes in hepatocytes that would otherwise be subject to permanent genome copy loss with hepatocellular cytokinesis.

\section{METHODS}

\section{Preparation of Recombinant Adeno-Associated Viral Vectors}

AAVrh10- and AAV9-luciferase are serotype rh10 and 9 vectors containing the firefly luciferase reporter gene and the chicken $\beta$-actin promoter/CMV enhancer that have been previously described in our laboratory (8). AAV was prepared by triple transfection of 293 cells as described previously (6). Viral titer was determined by quantitative real-time polymerase chain reaction.

\section{Animal Procedures}

Procedures were approved by the University of California, Los Angeles Committee on Animal Research. FVB/N female and male mice were purchased from Charles River Breeding Laboratories (Wilmington, MA). MyD88 knockout mice and C57BL/6 controls were from Jackson Laboratories (Bar Harbor, ME). FVB/N mice were used for all studies otherwise. At birth, an intravenous injection of $3 \times 10^{12}$ AAV genome copies $(\mathrm{gc}) / \mathrm{kg}$ in $50 \mu \mathrm{l}$ of normal saline was performed as previously described (6). Adult mice received alum/ luciferase by intraperitoneal injection to the right lower abdomen. AAV was delivered in adults as $3 \times 10^{12} \mathrm{gc} / \mathrm{kg}$ in $200 \mu \mathrm{l}$ of normal saline by tail vein injection.

Recombinant luciferase (Promega, Madison, WI) was mixed with a preformulated aqueous solution of aluminum hydroxide and magnesium hydroxide (Imject Alum, Pierce, Rockford, IL) in a 1:1 ratio according to the manufacturer's instructions. A volume of $200 \mu \mathrm{l}$ (including $1 \mu \mathrm{g}$ of recombinant luciferase) was administered intraperitoneally to each mouse.

\section{In Vivo Bioluminescent Imaging and Tissue Luminometry}

Mice were anesthetized and injected intraperitoneally with an aqueous solution of luciferin substrate and imaged as previously described (6). Tissue luminometry and normalization to protein concentrations were performed as previously described (38).

\section{ELISpot for IFN- $\boldsymbol{\gamma}$ and IL-2}

Mice were euthanized, spleens harvested aseptically, finely minced in $10 \mathrm{ml}$ RPMI, and filtered through a $40 \mu \mathrm{m}$ cell strainer to remove debris. The cell suspension was transferred to a $15-\mathrm{ml}$ tube and centrifuged at $200 \times \mathrm{g}$ for $5 \mathrm{~min}$. Cells were resuspended at $5 \times 10^{6}$ viable cells/ml in RPMI with $10 \%$ FBS and penicillin and streptomycin. Concavalin A (ConA), a nonspecific mitogen, was used as a positive control for the proliferative ability of splenocytes in the assay. Samples were set up in triplicate in a 96-well plate with $1 \mu \mathrm{g}$ recombinant luciferase (Promega) or ConA ( $1 \mu \mathrm{g}), 100 \mu \mathrm{l}$ of growth medium, and 100 $\mu \mathrm{l}$ of spleen cell suspension. Antibody pairs were used and performed per manufacturer instructions of analysis of interleuken-2 (IL-2) and interferon- $\gamma($ IFN- $\gamma$ ) (MabTech, Cincinnati, OH). Spots were analyzed by using an ImmunoSpot/BioSpot UV Analyzer (CTL Analyzers, Shaker Heights, $\mathrm{OH})$. Change as compared to unstimulated negative control cells was plotted.

\section{ELISA Assays}

ELISA for antiluciferase antibodies was performed as previously described (37). Positive control sera were obtained from serum samples of adult mice that had been injected with AAV-luciferase and had previously demonstrated antiluciferase antibody levels (4). Animals injected neonatally with AAV-luciferase $(n=5)$ were tested at each time point and the 1:20 dilution is presented in Figure 6.

ELISA for anti-AAV9 and rh10 antibodies. Ninety-six well plates were coated overnight at $4{ }^{\circ} \mathrm{C}$ with $1 \times 10^{9} \mathrm{gc}$ of AAVrh10 or AAV9 vector preparations per well in PBS. An ELISA was then performed as outlined (37). Positive control sera were obtained from
AAV-luciferase-injected adult mice with established anti-AAV antibody titers. AAV-injected $(n=5)$ and control animals $(n=5)$ were tested at each time point and the 1:5 dilution is presented in Figure 5.

\section{Genome Copy Number Determination}

At regular intervals, mice were euthanized and liver tissue was removed. Genomic DNA was extracted using a DNAEasy Kit (Qiagen, Germatown, MD) and quantitated by nanodrop (Implen, Westlake Village, CA) and real-time quantitative polymerase chain reaction performed as described (6).

\section{Statistical Calculations}

Mean, standard deviation, standard error of the mean, and Student's $T$-test were calculated using standard formulae. $T$-test was used for paired comparisons while the comparison of three groups was performed using analysis of variance (ANOVA). P values of $<0.05$ were considered significant.

\section{STATEMENT OF FINANCIAL SUPPORT}

This work was supported by grants to G.S.L. from the National Institutes of Health (NIH; 5K08HD057555-05, 1R01NS071076-05A1, and 1R01NS07107604S1). D.S.T. received funding from the Society of University Surgeons and was a recipient of a National Institute of General Medical Sciences Medical Genetics NIH T32 (GM008243). The Immuno/BioSpot Core is supported by UCLA Center for AIDS Research grant 5P30 AI028697 and the UCLA AIDS Institute. It is also supported by a grant from the James B. Pendleton Charitable Trust and a donation from the UCLA Children's Discovery and Innovation Institute.

Disclosures: No authors have declared a conflict of interest regarding this work.

\section{REFERENCES}

1. Saint-Remy JM. Immunology of factor VIII inhibitors. Semin Thromb Hemost 2002;28:265-8.

2. Fields PA, Arruda VR, Armstrong E, et al. Risk and prevention of antifactor IX formation in AAV-mediated gene transfer in the context of a large deletion of F9. Mol Ther 2001;4:201-10.

3. Herzog RW, Mount JD, Arruda VR, High KA, Lothrop CD Jr. Muscledirected gene transfer and transient immune suppression result in sustained partial correction of canine hemophilia B caused by a null mutation. Mol Ther 2001;4:192-200.

4. Fields PA, Kowalczyk DW, Arruda VR, et al. Role of vector in activation of $\mathrm{T}$ cell subsets in immune responses against the secreted transgene product factor IX. Mol Ther 2000;1:225-35.

5. Nathwani AC, Davidoff A, Hanawa H, Zhou JF, Vanin EF, Nienhuis AW. Factors influencing in vivo transduction by recombinant adeno-associated viral vectors expressing the human factor IX cDNA. Blood 2001;97:1258-65.

6. Hu C, Busuttil RW, Lipshutz GS. RH10 provides superior transgene expression in mice when compared with natural AAV serotypes for neonatal gene therapy. J Gene Med 2010;12:766-78.

7. Hu C, Lipshutz GS. AAV-based neonatal gene therapy for hemophilia A: long-term correction and avoidance of immune responses in mice. Gene Ther 2012;19:1166-76.

8. Hu C, Kasten J, Park H, et al. Myocyte-mediated arginase expression controls hyperargininemia but not hyperammonemia in arginase-deficient mice. Mol Ther 2014;22:1792-802.

9. Kok CY, Cunningham SC, Carpenter KH, et al. Adeno-associated virusmediated rescue of neonatal lethality in argininosuccinate synthetasedeficient mice. Mol Ther 2013;21:1823-31.

10. Chirmule N, Xiao W, Truneh A, et al. Humoral immunity to adeno-associated virus type 2 vectors following administration to murine and nonhuman primate muscle. J Virol 2000;74:2420-5.

11. Moskalenko M, Chen L, van Roey M, et al. Epitope mapping of human anti-adeno-associated virus type 2 neutralizing antibodies: implications for gene therapy and virus structure. J Virol 2000;74:1761-6.

12. Halbert CL, Standaert TA, Wilson CB, Miller AD. Successful readministration of adeno-associated virus vectors to the mouse lung requires transient immunosuppression during the initial exposure. J Virol 1998;72: 9795-805. 


\section{Articles Tai etal.}

13. Manning WC, Zhou S, Bland MP, Escobedo JA, Dwarki V. Transient immunosuppression allows transgene expression following readministration of adeno-associated viral vectors. Hum Gene Ther 1998;9:477-85.

14. Iwamoto HS. The window of opportunity for fetal gene therapy. Mol Hum Reprod 1996;2:472-4.

15. Lipshutz GS, Sarkar R, Flebbe-Rehwaldt L, Kazazian H, Gaensler KM. Short-term correction of factor VIII deficiency in a murine model of hemophilia A after delivery of adenovirus murine factor VIII in utero. Proc Natl Acad Sci USA 1999;96:13324-9.

16. Mitchell M, Jerebtsova M, Batshaw ML, Newman K, Ye X. Long-term gene transfer to mouse fetuses with recombinant adenovirus and adenoassociated virus (AAV) vectors. Gene Ther 2000;7:1986-92.

17. Nelson WE. Growth and development in the infant and child. In Nelson WE (ed.) Textbook of Pediatrics. W.B Saunders Company: Philadelphia, 1964. pp. 21.

18. Coppoletta JM, Wolbach SB. Body length and organ weights of infants and children: a study of the body length and normal weights of the more important vital organs of the body between birth and twelve years of age. Am J Pathol 1933;9:55-70.

19. Wang L, Bell P, Lin J, Calcedo R, Tarantal AF, Wilson JM. AAV8-mediated hepatic gene transfer in infant rhesus monkeys (Macaca mulatta). Mol Ther 2011;19:2012-20.

20. Cunningham SC, Dane AP, Spinoulas A, Logan GJ, Alexander IE. Gene delivery to the juvenile mouse liver using AAV2/8 vectors. Mol Ther 2008;16:1081-8.

21. Zhou S, Kurt-Jones EA, Mandell L, et al. MyD88 is critical for the development of innate and adaptive immunity during acute lymphocytic choriomeningitis virus infection. Eur J Immunol 2005;35:822-30.

22. Zhou S, Kurt-Jones EA, Cerny AM, Chan M, Bronson RT, Finberg RW. MyD88 intrinsically regulates CD4 T-cell responses. J Virol 2009;83:1625-34.

23. Smith KA. Interleukin-2: inception, impact, and implications. Science 1988;240:1169-76.

24. Schroder K, Hertzog PJ, Ravasi T, Hume DA. Interferon-gamma: an overview of signals, mechanisms and functions. J Leukoc Biol 2004;75:163-89.
25. Ohashi PS, Oehen S, Buerki K, et al. Ablation of "tolerance" and induction of diabetes by virus infection in viral antigen transgenic mice. Cell 1991;65:305-17.

26. Adkins B. T-cell function in newborn mice and humans. Immunol Today 1999;20:330-5.

27. Piguet PF, Irle C, Kollatte E, Vassalli P. Post-thymic T lymphocyte maturation during ontogenesis. J Exp Med 1981;154:581-93.

28. Ridge JP, Fuchs EJ, Matzinger P. Neonatal tolerance revisited: turning on newborn T cells with dendritic cells. Science 1996;271:1723-6.

29. Bogue M, Candéias S, Benoist C, Mathis D. A special repertoire of alpha:beta T cells in neonatal mice. EMBO J 1991;10:3647-54.

30. Forsthuber T, Yip HC, Lehmann PV. Induction of TH1 and TH2 immunity in neonatal mice. Science 1996;271:1728-30.

31. Garza KM, Agersborg SS, Baker E, Tung KS. Persistence of physiological self antigen is required for the regulation of self tolerance. J Immunol 2000;164:3982-9.

32. Rocha B, Tanchot C, Von Boehmer H. Clonal anergy blocks in vivo growth of mature T cells and can be reversed in the absence of antigen. J Exp Med 1993;177:1517-21.

33. Triplett EL. On the mechanism of immunologic self recognition. J Immunol 1962;89:505-10.

34. Lipshutz GS, Gruber CA, Cao Y, Hardy J, Contag CH, Gaensler KM. In utero delivery of adeno-associated viral vectors: intraperitoneal gene transfer produces long-term expression. Mol Ther 2001;3:284-92.

35. Fausto N, Campbell JS. The role of hepatocytes and oval cells in liver regeneration and repopulation. Mech Dev 2003;120:117-30.

36. Magami Y, Azuma T, Inokuchi $\mathrm{H}$, et al. Cell proliferation and renewal of normal hepatocytes and bile duct cells in adult mouse liver. Liver 2002;22:419-25.

37. Lipshutz GS, Flebbe-Rehwaldt L, Gaensler KM. Reexpression following readministration of an adenoviral vector in adult mice after initial in utero adenoviral administration. Mol Ther 2000;2:374-80.

38. Nguyen AT, Dow AC, Kupiec-Weglinski J, Busuttil RW, Lipshutz GS. Evaluation of gene promoters for liver expression by hydrodynamic gene transfer. J Surg Res 2008;148:60-6. 